\title{
Valproic acid and immune thrombocytopenia
}

\author{
RONALD D BARR, SHIRLEY A COPELAND, MICHELLE L STOCKWELL, \\ NEIL MORRIS, AND JOHN C KELTON
}

\begin{abstract}
Department of Pediatrics, Department of Pathology, and Department of Medicine, McMaster University, Hamilton, Ontario, Canada
\end{abstract}

SUMMARY During the course of a prospective serial study over a period of one year, findings compatible with immune-mediated thrombocytolysis accompanied the administration of valproic acid in about half of 45 children with epilepsy. Thrombocytopenia occurred in 15 and neutropenia in 12 patients, but was transient and self-limiting in each. The association is probably one of cause and effect, and it may reflect the structural similarity between valproic acid and constituents of cell membranes.

Circumstantial evidence suggests that thrombocytopenia may result from the administration of valproic acid in clinical practice. ${ }^{1-6}$ From recent case studies we have surmised that more than one pathogenetic mechanism may exist since, in one patient, thrombocytopenia resolved despite continuing drug administration. ${ }^{7}$ Thus, in some instances this association may not be one of cause and effect, but rather may reflect the induction of thrombocytopenia by coincidental viral infection. ${ }^{78}$ With a view to resolving these issues, we embarked on a prospective investigation of a group of children who were receiving treatment with valproic acid.

\section{Study design}

All patients who were being given valproic acid under the supervision of a single paediatrician (N M) were considered to be eligible for study. At intervals of about 2 months during the period January to December 1980, each patient underwent serial sampling of venous blood on routine monitoring for determination of the following indices by standard methods: haemoglobin concentration; reticulocyte count; direct antiglobulin test; total and differential leucocyte counts; platelet counts; serum albumin; total globulins; immunoglobulins $\mathbf{G}, \mathbf{A}$, and $\mathbf{M}$; and serum valproic acid concentration. Concentrations of haemoglobin, leucocytes, and immunoglobulins were compared with age-related control values. Platelet associated IgG (PA IgG) was measured by the antiglobulin consumption assay ${ }^{9}$ (normal values $\gtrless 10$ femtograms IgG per platelet). Statistical analyses were performed by the Wilcoxon signed rank test and linear regression as appropriate.

\section{Results}

Patients. Forty-five patients with epilepsy (28 boys, 17 girls) aged between 0.5 and 20 (median age 6) years were recruited to the study. Nine patients entered at the time of initiation of treatment with vaproic acid and all their haematological indices were normal. The remainder had been receiving vaproic acid for 1-136 (median 38) weeks before entry. In 12 patients additional anticonvulsant agents were used: 5 received carbamazepine, 4 phenobarbitone, 2 phenytoin, 2 phenobarbitone plus phenytoin, and 1 was given ACTH. Patients were studied on 1-7 occasions with a median of 4 samples being obtained from each.

Erythrocyte indices. Only one patient became anaemic; a 14-year-old girl had a nadir of haemoglobin concentration of $8 \cdot 8 \mathrm{~g} / \mathrm{dl}$ with a spontaneous return to normal. A diagnosis of transient erythroblastopenia of childhood was made (see below). No other patient had an absolute reticulocytosis and no positive direct antiglobulin test was recorded.

Serum proteins. All measurements of serum albumin and globulin concentrations were normal (greater than $15 \mathrm{~g} / 1$ and $35 \mathrm{~g} / 1$ respectively). One child with transient erythroblastopenia of childhood (see above) had a total globulin concentration of $44 \mathrm{~g} / 1$ with an IgG assay of $25 \cdot 2 \mathrm{~g} / 1$. No other patient showed an increase in total globulins. Only one other patient had an abnormal immunoglobulin profile, an 11-year-old boy with selective IgA deficiency $(0 \cdot 17 \mathrm{~g} / \mathrm{l})$ and no stigmata of the Wiscott-Aldrich syndrome. 
Leucocyte profile. Although the total leucocyte concentration was normal in all samples, 12 of 45 patients developed absolute neutropenia at some time during the study including 2 of 12 patients receiving more than one anticonvulsant. In all cases this phenomenon was transient and self-limiting.

Platelet counts and PA IgG. One-third of the population (15 of 45) were observed to be thrombocytopenic (platelet count less than $150 \times 10^{9} / 1$ ) at some time during the study (range of lowest platelet counts: $35-149 \times 10^{9} / 1$ ), including 4 of 12 patients receiving more than one anticonvulsant (Tables 1-3). This was transient and self-limiting in all cases. The relationship between platelet count and PA IgG is illustrated in the Figure. In $82 \%$ of instances thrombocytopenia was associated with an increased PA IgG, and there was a significant inverse correlation $(\mathrm{P}<0.05)$ between the platelet count and the level of PA IgG (13-24 femtograms IgG per platelet).

At least $40 \%$ of the patients had increases in PA IgG at some time during the study and $65 \%$ of these were accompanied by normal platelet counts. However, the counts $\left(53-354 \times 10^{9} / 1\right.$, median $\left.173 \times 10^{9} / 1\right)$ of those samples with raised PA IgG determinations were significantly lower $(P<0.005)$ than those which were associated with normal levels of PA IgG (144-509 $\times 10^{9} / 1$, median $\left.240 \times 10^{9} / 1\right)$.

Valproic acid concentrations*. There was no significant difference in valproic acid levels between samples with thrombocytopenia (valproic acid 18-115, median $95 \mathrm{mg} / \mathrm{l}$ ) and those with normal platelet counts (valproic acid 11-136, median

* Valproic acid $144 \mathrm{mg}$ per litre $=1.0 \times 10^{-3} \mathrm{molll}$.

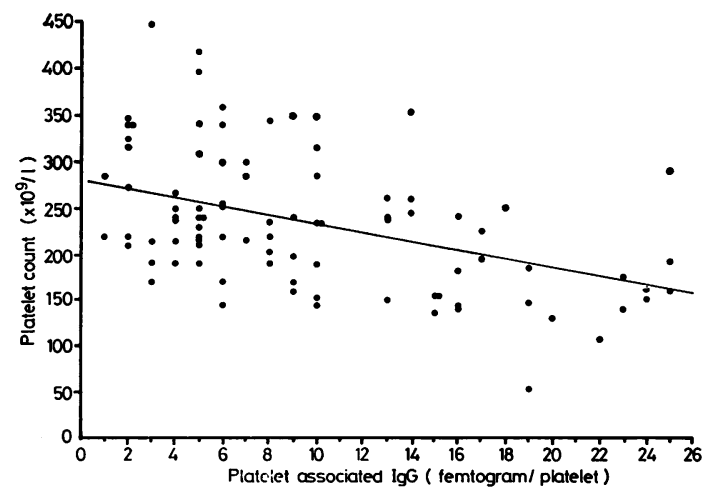

Figure Relationship between the concentration of plateiet-associated $\operatorname{Ig} G(x)$ and the platelet count $(y)$ when $x$ is expressed as femtograms per platelet and $y$ as the number $\left(\times 10^{9}\right)$ per litre of blood, $y=-5 \cdot 287 x+286 \cdot 6$. This is a significant inverse correlution, $P<0.001$.
Table 1 Patients studied during treatment with valproic acid alone

\begin{tabular}{llc}
\hline Case & Platelet nadir $(\times 109 / l)$ & $\begin{array}{c}\text { Interval }^{*} \\
(\text { months })\end{array}$ \\
\hline 1 & 397 & 8 \\
2 & 143 & 16 \\
5 & 175 & 30 \\
6 & 220 & 15 \\
7 & 190 & 9 \\
9 & 260 & 18 \\
13 & 147 & 17 \\
14 & 96 & 16 \\
15 & 161 & 16 \\
16 & 210 & 14 \\
18 & 242 & 14 \\
19 & 140 & 140 days \\
20 & 35 & 110 days \\
21 & 240 & 9 \\
22 & 255 & 9 \\
25 & 179 & 40 days \\
26 & 145 & 110 days \\
27 & 149 & 8 \\
28 & 234 & 15 days \\
31 & 242 & 7 \\
34 & 169 & 24 \\
35 & 135 & 6 days \\
37 & 124 & 24 \\
42 & 213 & \\
\hline
\end{tabular}

*Interval between initiation of treatment and platelet nadir.

Table 2 Patients studied during combination anticonvulsant treatment

\begin{tabular}{rlc}
\hline Case & Platelet nadir $\left(\times 10^{9} / l\right)$ & $\begin{array}{l}\text { Interval } \\
(\text { months })\end{array}$ \\
\hline 3 & 317 & 13 \\
4 & 147 & 22 \\
8 & 345 & 15 days \\
10 & 170 & 8 \\
11 & 130 & 13 \\
12 & 144 & 6 \\
17 & 266 & 140 days \\
24 & 134 & 65 days \\
32 & 170 & 90 days \\
38 & 313 & 25 \\
43 & 269 & 115 days \\
45 & 208 & 30 days \\
\hline
\end{tabular}

*Interval between initiation of treatment with valproic acid and platelet nadir.

Table 3 Patients studied before and during treatment with valproic acid

\begin{tabular}{llll}
\hline Case & Platelet $\left(\times 10^{9} / l\right)$ & $\begin{array}{l}\text { Interval } \\
(\text { days })\end{array}$ \\
\cline { 2 - 4 } & Count before treatment & Nadir during treatment & \\
\hline 23 & 215 & 144 & 75 \\
29 & 250 & 210 & 60 \\
30 & 345 & 325 & 25 \\
33 & 260 & 102 & 120 \\
36 & 259 & 230 & 30 \\
39 & 284 & 215 & 20 \\
40 & 354 & 170 & 40 \\
41 & 240 & 198 & 30 \\
44 & 253 & 240 & 60 \\
\hline
\end{tabular}

*Interval between initiation of treatment and platelet nadir. 
$88 \mathrm{mg} / \mathrm{l})$. In 5 additional samples no valproic acid was identified. Furthermore, the drug concentrations were no greater in samples with raised PA IgG levels (valproic acid 0-150, median $95 \mathrm{mg} / \mathrm{l}$ ) than in those with normal levels of PA IgG (valproic acid 0-141, median $88 \mathrm{mg} / \mathrm{l})$.

\section{Discussion}

Valproic acid is chemically unique among the anticonvulsant drugs, being a branched chain carboxylic acid, ${ }^{10}$ and it is structurally similar to the fatty acid constituents of cell membranes. ${ }^{5}$ This may afford an explanation for the impact of the drug not only on platelet numbers but also on platelet function. ${ }^{6} 1112$ While the clinical importance of platelet dysfunction induced by valproic acid is unknown, thrombocytopenia associated with the administration of this agent may be severe. ${ }^{6}$

In a recent retrospective study of nearly 1500 samples from mentally handicapped patients, Eastham and Jancar ${ }^{13}$ found no difference in the platelet counts of individuals with and without epilepsy who were receiving anticonvulsants other than valproic acid, but patients with epilepsy who were receiving valproic acid had significantly lower counts than those who were being treated with other drugs. Serial, prospective sampling, as conducted in the present study, shows that such thrombacytopenia, while common, is always transient and self-limiting despite continuing drug administration; we had elected to pursue this policy on the basis of previous clinical experience ${ }^{7}$ of the resolution of thrombocytopenia without interruption of treatment with valproic acid. Moreover, the degree of thrombocytopenia is such that a haemorrhagic diathesis is predictably unlikely. Similar observations have been made by Wilder (personal communication) in a group of 25 adolescents and young adults.

Sandler et al.$^{14}$ have suggested that valproic acid may evoke the occasional production of an antibody directed against circulating platelets, even in the absence of thrombocytopenia. Our data, obtained with a much more sensitive technique, indicate that nearly half of patients who were receiving valproic acid had an increased level of PA IgG at some time during the study. Although $65 \%$ of these samples had a normal platelet count, it is likely that they reflected a compensated thrombocytolytic state, with increased platelet production matching increased platelet destruction, since samples with raised PA IgG determinations had significantly lower platelet counts than had those with normal levels of PA IgG. Thus, immune platelet destruction may occur more frequently in association with the administration of valproic acid than is suggested by the incidence of frank thrombocytopenia. A formal study of platelet survival would be necessary to clarify this prospect.

Transient, self-limiting neutropenia was also a common finding during the present study in which no florid clinical stigmata of infection were apparent. Furthermore, in only 3 children were thrombocytopenia and neutropenia recorded at the same time. Similar experience has been recorded previously ${ }^{15}$ and anecdotal evidence points to a cause and effect relationship with administration of valproic acid. ${ }^{16}$ However, we could find no association between the concentration of valproic acid in the blood and either the platelet count or the level of PA IgG. At first sight these findings might negate a causal association between drug administration and thrombocytopenia, but from pharmacokinetic data it is clear that blood levels of valproic acid are labile and unpredictable. ${ }^{10}$ Moreover, although in patients with drug-induced thrombocytopenia the platelet count typically remains reduced while the drug continues to be taken, transient and selflimiting thrombocytopenia has been described in association with the use of heparin. ${ }^{17} 18$

Thus, immune-mediated thrombocytopenia may be a common accompaniment of the administration of valproic acid, but the pathogenesis of this phenomenon remains to be explored.

We thank Mrs $\mathbf{J}$ Moore for performing the assays of PA IgG and Mr T Bailey, Mrs B Roberge, and Mrs C Williams for technical assistance.

These studies were supported by grants from the Medical Research Council of Canada.

\section{References}

1 Sutor A H, Jesdinsky-Buscher C. Gerinnungsveranderungen durch Dipropylessigsaure Ergenyl. Med Welt 1974; 25: 447-9.

2 Von Voss H, Petrich C, Karch D, Schulz H U, Gobel U. Letter: Sodium valproate and platelet function. $\mathrm{Br} \mathrm{Med} J$ 1976; ii: 179.

3 Winfield D A, Benton P, Espir M L E, Arthur L J H. Sodium valproate and thrombocytopenia. Br Med J 1976; ii: 981 .

4 Raworth R E, Birchall G. Letter: Sodium valproate and platelet count. Lancet 1978; i : 670-1.

5 Sandler R M, Emberson C, Roberts G E, Voak D, Darnborough J, Heeley A F. IgM platelet auto-antibody due to sodium valproate. $B r$ Med J 1978; ii : 1683-4.

6 Von Voss H, Petrich C, Schulz H U, Karch D, Göbel U. Hemorrhagic diathesis and platelet dysfunction in children under treatment with sodium valproate. Epilepsia 1978 ; 19: 113-4.

7 Morris N, Barr R D, Pai K R M, Kelton J G. Valproic acid and thrombocytopenia. Can Med Assoc J 1981; 125: 63-4.

8 Cole A P. Transient thrombocytopenia in a child on 
sodium valproate. Dev Med Child Neurol 1978; 20: 487-90.

9 Kelton J G, Neame P B, Bishop J, Ali M, Gauldie J, Hirsh J. The direct assay for platelet associated IgG (PA IgG): lack of association between antibody level and platelet size. Blood 1979; 53: 73-80.

10 Bruni J, Wilder B J. Valproic acid-review of a new anti-epileptic drug. Arch Neurol 1979; 36: 393-8.

11 Richardson S G N, Fletcher D J, Jeavons P M, Stuart J. Letter: Sodium valproate and platelet function. $\mathrm{Br} \mathrm{Med} J$ 1976; i: 221-2.

12 Beran R G, Read T, Reid M, et al. Individualised patient assessment in the treatment of epilepsy with sodium valproate. Epilepsia 1980; 21 : 379-86.

13 Eastham R D, Jancar J. Letter: Sodium valproate and platelet counts. $\mathrm{Br}$ Med J 1980; 280: 186.

14 Sandler P M, Bevan P C, Roberts G E, et al. Interaction between sodium valproate and platelets: a further study. Br Med J 1979; ii : 1476.

15 Coulter D L, Wu H, Allen R J, Valproic acid therapy in childhood epilepsy. JAMA 1980; 244: 785-8.
16 Jaeken J, Van Goethem C, Casaer P, Devlieger H, Eggermont E, Pilat M. Letter: Neutropenia during sodium valproate treatment. Arch Dis Child 1979; 54: 986-7.

17 Kelton J G, Powers P J. Heparin-associated thrombocytopenia: an immune disorder. In: Lundblad $\mathbf{R} \mathbf{L}$, Brown W V, Mann K G, Roberts H R, eds. Chemistry and biology of heparin. New York: Elsevier-North Holland, 1980: 365-75.

18 Powers P J, Cuthbert D, Hirsh J. Thrombocytopenia found uncommonly during heparin therapy. JAMA 1979; 241 : 2396-7.

Correspondence to Dr R D Barr, Room 3N27D, McMaster University Medical Centre, 1200 Main Street West, Hamilton, Ontario, Canada L8N $3 Z 5$.

Received 12 May 1982 